\title{
Level of Anxiety and Depression in Cardiac Syndrome X
}

\author{
Elif Ijlal Cekirdekci Baris Bugan \\ Department of Cardiology, Dr. Suat Gunsel University of Kyrenia Hospital, Kyrenia, Cyprus
}

\section{Significance of the Study}

- In our study, cardiac syndrome X has been found to be associated with depression, anxiety, and somatic concern; thus, it seems reasonable that improving quality of life and psychological support may benefit patients with cardiac syndrome $\mathrm{X}$. This study may provide a new perspective on this group of patients and may encourage further prospective studies in larger populations.

\section{Keywords}

Anxiety $\cdot$ Cardiac syndrome X·Depression

\section{Abstract}

Objective: Cardiac syndrome X (CSX) is defined as anginalike symptoms, abnormalities on stress testing, and normal epicardial coronary arteries on coronary angiography. The aim of this study was to determine the Hospital Anxiety and Depression scores of patients with CSX and to compare with healthy controls. Materials/Subjects and Methods: Patients undergoing coronary angiography between January 2015 and December 2016 because of clinical indications, including abnormal noninvasive test results were examined. Two hundred and 10 subjects (110 patients with CSX, 100 controls) were enrolled. Demographic characteristics including age, education level, marriage status, and history of stressful life events were recorded. The Turkish version of the Hospital Anxiety and Depression scale was evaluated in the study population. Results: Anxiety, depression, and total scores in the patients with CSX were significantly higher than those in the control group $(p<0.001, p<0.003, p<0.001$, respectively). Among women, anxiety, depression, total scores, and stressful life events were significantly higher in the CSX group ( $p=0.006, p=0.015, p=0.001, p<0.001$, respectively). Patients with lower educational status had higher anxiety scores $(p=0.03)$, stressful life events, and HAD-A >10 were the only independent predictors of CSX in logistic regression analysis with comparable ORs $2.256(95 \% \mathrm{Cl} 1.057-4.817$, $p=0.03)$ and $2,399(95 \% \mathrm{Cl} 1.248-4.613, p=0.009)$ respectively. Conclusion: The results of our research suggest that patients with CSX have a high prevalence of stress and psychiatric disturbances. Interventions targeted toward improving the quality of life and to give psychological support may have the potential benefits especially for women and individuals with lower education. @ 2018 The Author(s)

Published by S. Karger AG, Basel

\section{Introduction}

Chest pain is the typical sign of ischemic heart disease; however, up to $20-30 \%$ of patients demonstrate no signs of obstructive coronary artery disease [1]. The most common causes of non-cardiac chest pain are esophageal disorders (gastro-esophageal reflux and esophageal dys-

\begin{tabular}{ll}
\hline KARGER & $\begin{array}{l}\text { ( 2018 The Author(s) Karger } \\
\text { Published by S. Karger AG, Basel Open cacess }\end{array}$ \\
E-Mail karger@karger.com & $\begin{array}{l}\text { This is an Open Access article licensed under the Creative Commons } \\
\text { Attribution-NonCommercial-4.0 International License (CC BY-NC) } \\
\text { (http://www.karger.com/Services/OpenAccessLicense), applicable to } \\
\text { the online version of the article only. Usage and distribution for com- } \\
\text { mercial purposes requires written permission. }\end{array}$
\end{tabular}

Elif Ijlal Cekirdekci, MD, Assist Prof Department of Cardiology

Dr. Suat Gunsel University of Kyrenia Hospital

Sehit Yahya Bakır Street-10, Karakum, Kyrenia (Cyprus)

E-Mail elifcekirdekci@ hotmail.com 
motility), musculoskeletal disorders (costochondritis and increased muscular tension), and psychological problems (panic attacks and depression) [2]. Diagnostic uncertainty for persistent chest pain is associated with adverse quality of life, disability, and health care costs.

Although Cardiac Syndrome X (CSX) was first described by Kemp [3] in 1973 as typical chest pain with angiographically normal epicardial coronaries and positive stress tests for myocardial ischemia, recent studies have contributed to a more precise definition using the term microvascular angina. The diagnosis of CSX is primarily based on the exclusion of other non-cardiac chest pain. Coronary microvascular dysfunction, perfusion abnormalities and abnormal visceral pain perception have been suggested to be the mechanism of this clinical entity $[4,5]$. Microvascular angina is characterized by a transient myocardial ischemia secondary to the dysfunction of the resistant coronary vessels $(<500 \mu \mathrm{m})$, which are not visualized in coronary angiography $[6,7]$.

Autonomic disturbances with persisting sympathetic stimulation promotes psychological abnormalities particularly anxiety disorders. Increased sympathetic stimulation may be the reason for a positive stress test(s) for myocardial ischemia. Improved responses with $\beta$ blockade, and improved coronary flow reserve with a blockade, and abnormal reactivity to vasoconstrictor mediators in arteriolar resistance vessel support this conclusion [8]. Cunningham et al. [9] showed the significant positive effects of transcendental meditation on objective exercise test variables and quality of life in CSX.

Previous studies have pointed out higher levels of anxiety especially among women with CSX when compared to healthy individuals and to those with confirmed coronary heart disease $[10,11]$. Therefore, imipramine, an analgesic with an independent antidepressant effect, and techniques for relaxation and stress reduction have been used to reduce chest pain symptoms in this group of patients $[12,13]$.

The aim of the present study was to determine the Hospital Anxiety and Depression scores of patients with CSX and to compare these scores with healthy controls. In accordance with this aim, the research questions for this study were as follows: (1) Is there a relationship between presence of depression and anxiety disorders as determined by a score on the Hospital Anxiety and Depression Scale (HADS) and CSX? (2) Do scores on the HADS differentiate between CSX and control group? (3) Do scores on the HADS differ between men and women? (4) Is there a relationship between educational status and experience of stressful life events among the CSX group?

\section{Materials and Methods}

Patients undergoing coronary angiography between January 2015 and December 2016 because of clinical indications, including symptoms of angina and abnormal noninvasive test results (either treadmill exercise tests or myocardial perfusion scintigraphy) were examined.

One hundred ten patients (52 men and 58 women, mean age: $52.5 \pm 10.8$ years) with abnormal noninvasive test results for myocardial ischemia and angiographically normal coronary arteries were enrolled as part of the CSX group. Age-matched 100 control subjects (41 men and 59 women, mean age: $52.4 \pm 9.7$ years) with no previous history of chronic illness were recruited from outpatient clinic. Demographic and clinical characteristics including age, education level, and marriage status were recorded.

The list of "threatening experiences" is a list of 12 life events that have been demonstrated to have a marked or moderate effect on psychological well-being [14]. This list was used to detect whether the participants experienced stressful life events. Each question has a yes/no response format. If the participants expressed one or more stressful life events, it was accepted that they experienced stressful life event(s). Additional self-reported experiences were also accepted.

Written informed consent was obtained from all the participants prior to enrollment. The Institutional Ethics Committee approved the study protocol, and the study was conducted in accordance with the Declaration of Helsinki. Exclusion criteria included the following: refusal to participate in the study and presence of unstable ischemic conditions (unstable angina pectoris and myocardial infarction), valvular heart disease, other systemic gastrointestinal, neurological, endocrine, psychiatric diseases that could affect functional and psychological status and psychiatric drug usage.

The HADS is designed to screen for the presence of depression and anxiety disorders in medically ill patients. The Turkish version of HADS, which was translated and validated by Aydemir et al. [15], was administered to the participants of the study. This self-administered 14-item questionnaire is split into 2 sub-scales (7 for anxiety and 7 for depression) using a 4-point Likert scale (ranging from 0 to 3 ). According to the Turkish version of HADS, the cutoff points for the anxiety and the depression are 10 and 7 respectively. After administering the HADS questionnaire, the anxiety score (HAD-A) and the depression score (HAD-D) were assessed.

\section{Statistics}

Statistical analyses were performed using SPSS software version 22 (SPSS Inc., Chicago, IL, USA). Subjects were grouped into CSX and control group. The variables were investigated using visual (histograms, probability plots) and analytical (KolmogorovSmirnov/Shapiro-Wilk test) methods to determine whether or not they are normally distributed. Continuous measurements were summarized as mean and $\mathrm{SD}$, whereas categorical variables were summarized as number of cases with percentage (\%). Overall comparisons of categorical variables were performed using chi-square test. Student $t$ test was used for normally distributed parameters. A logistic regression was run to determine the ability to predict a patient's probability of CSX based on age, marriage status, educational level, experience of stressful life events, and HAD-A $>10$ and HAD-D $>7$. 
Hosmer-Lemeshow goodness of fit statistics were used to assess model fit. The capacity of HAD-A and HAD-D scores in predicting CSX were analyzed using (ROC) curve analysis. When a significant cutoff value was observed, the sensitivity, specificity, positive, and negative predictive values were presented. While evaluating the area under the curve, a 5\% type-I error level was used to show statistical significant predictive value of the test variables.

\section{Results}

Demographic characteristics of the study population are summarized in Table 1. There were no statistically significant differences between the groups in terms of age, gender, marital status, and education level.

Total hospital anxiety and depression scores of the patients in the study were $16.6 \pm 8.25$ (the mean HAD$\mathrm{D}$ was $6.73 \pm 4.34$ and mean HAD-A was 9.89 \pm 5.65$)$. CSX patients had higher anxiety and total scores than healthy controls $(11.2 \pm 5.48$ vs. $8 \pm 5.35, p<0.001,18.8$ \pm 8.03 vs. $13.5 \pm 7.62, p<0.001$ respectively). CSX patients had higher depression scores than the healthy controls $(6.55 \pm 3.99$ vs. $5.58 \pm 3.76, p=0.003)$. Clinical levels of anxiety $(>10)$ were found in $56.4 \%(n=62)$ of Syndrome X patients compared with $34 \%(n=34)$ of healthy controls $(p<0.01)$. Clinical levels of depression $(>7)$ were found in $47.3 \%(n=52)$ of Syndrome X patients compared with $30 \%(n=30)$ of healthy controls $(p=0.019)$. Stressful life events are statistically higher in the CSX group than those in the control group $(31.8$ vs. $17 \%, p=0.03$ )

Of the 110 patients with CSX, 58 (52.7\%) were female, whereas $47(59.5 \%)$ of the control group were female. Among women, HAD-A, HAD-D, total scores and stressful life events were higher in the CSX group compared with those in the control group $(p=0.006, p=0.015, p=$ $0.001, p<0.001$ respectively), whereas no statistically significant differences were found in terms of marriage and educational status between groups (Table 1). Of the 110 patients with CSX, $52(47.3 \%)$ were men, whereas 41 (41\%) of the control group were men. Anxiety and total scores were significantly higher in the CSX group compared with those of the control group (11.2 \pm 5.48 vs. $8 \pm$ $5.35, p=0.01,18.3 \pm 7.97$ vs. $13.3 \pm 7.97, p<0.001$, respectively), whereas no statistically significant differences were found in terms of depression scores, stressful life events, marriage, and educational status between groups (Table 1).

A negative association was found between education level and experience of recent stressful events in the CSX group $(p=0.038)$.
Table 1. Baseline characteristics of patients

\begin{tabular}{|c|c|c|c|}
\hline & $\begin{array}{l}\text { CSX } \\
(n=110)\end{array}$ & $\begin{array}{l}\text { Control } \\
(n=100)\end{array}$ & $\begin{array}{l}p \\
\text { value }\end{array}$ \\
\hline Age, years, mean $\pm S D$ & $52.56 \pm 10.8$ & $52.41 \pm 9.77$ & 0.91 \\
\hline Gender, $n(\%)$ & & & 0.37 \\
\hline Female & $58(52.7)$ & $59(59)$ & \\
\hline Male & $52(47.3)$ & $41(41)$ & \\
\hline \multicolumn{4}{|l|}{ Marital status, $n(\%)$} \\
\hline Married & $81(73.6)$ & $68(68)$ & \\
\hline Single/widowed & $29(26.4)$ & $32(32)$ & 0.42 \\
\hline \multicolumn{4}{|l|}{ Education level, $n(\%)$} \\
\hline $1-8$ years & $30(27.3)$ & $23(23)$ & \\
\hline 9-12 years & $52(47.3)$ & $54(54)$ & 0.96 \\
\hline$>12$ years & $28(25.5)$ & $23(23)$ & \\
\hline Recent stressful life events & $35(31.8)$ & $17(17)$ & 0.038 \\
\hline $\mathrm{HAD}-\mathrm{A}>10, n(\%)$ & $62(56.4)$ & $34(34)$ & 0.003 \\
\hline $\mathrm{HAD}-\mathrm{D}>7, n(\%)$ & $52(47.3)$ & $30(30)$ & 0.019 \\
\hline
\end{tabular}

CSX, cardiac syndrome X; HAD-A, hospital anxiety and depression - anxiety score; HAD-D, hospital anxiety and depression - depression score.

Table 2. A logistic regression analysis to predict patient's probability of CSX

\begin{tabular}{llll}
\hline & OR & $\begin{array}{l}\text { HR } \\
(95 \% \mathrm{CI})\end{array}$ & $\begin{array}{l}p \\
\text { value }\end{array}$ \\
\hline Age, years & 1.009 & $0.973-1.047$ & 0.62 \\
Gender (men vs. women) & 1.467 & $0.775-2.780$ & 0.24 \\
Marital status (married vs. single) & 0.808 & $0.601-2.428$ & 0.59 \\
Education level (per year of school) & 0.924 & $0.332-2.570$ & 0.88 \\
Stressful life event & 2.256 & $1.057-4.817$ & 0.036 \\
HAD-A $>10$ & 2,399 & $1.248-4.613$ & 0.009 \\
HAD-D $>7$ & 1.491 & $0.771-2.884$ & 0.23
\end{tabular}

CSX, cardiac syndrome X; HR, hazard ratio.

In the ROC curve analysis, a HAD-A cutoff value of 10 with the sensitivity of $74 \%$ and the specificity of $81.2 \%$, positive predictive value of $79.5 \%$, negative predictive value of $75.7 \%$ and accuracy of $77.5 \%$ for the prediction of CSX (AUC 0.802, $p<0.001$ ) and a HAD-D cut-off value of 7 with the sensitivity of $71.4 \%$ and specificity $73.6 \%$, positive predictive value of $72.4 \%$, negative predictive value of $71.5 \%$ and accuracy of $72 \%$ for the prediction of CSX (AUC 0.761, $p<0.003$ ).

Stressful life events (OR 2.256, 95\% CI 1.057-4.817, $p=0.03)$ and HAD-A $>10$ (OR 2,399, 95\% CI 1.248 $4.613, p=0.009$ ) were the only independent predictors of CSX in logistic regression analysis with ORs (Table 2). 


\section{Discussion}

According to the present study, anxiety, depression, and total scores in the patients with CSX were significantly higher when compared to those of the control group. The results of our study showed that patients with CSX have high prevalence of stress and psychiatric disturbances.

CSX was considered to be a benign medical condition when compared to coronary artery disease [16-18]. However, this observation has been challenged by recent evidence. Studies have shown association with future cardiovascular events including myocardial infarction, hospitalization for heart failure, stroke, cardiac, and all-cause mortality, poor social and occupational prognosis, and ineffective therapeutic interventions $[19,20]$.

At the present time, most accepted mechanisms of this clinical entity are coronary microvascular dysfunction (i.e., abnormal dilatory responses and/or increased vasoconstriction); and enhanced sensitivity to cardiac pain or the so-called sensitive heart syndrome [21]. Most patients with CSX demonstrate emotional disorders and complaints incompatible with objective findings of coronary angiography [22]. Investigations have identified a reliable link between the development of CSX and psychosocial factors. CSX patients reported more depression, anxiety, and somatic concerns than patients with confirmed coronary disease [23]. In contrast to Lutfi's study [24], which demonstrated that the Taylor Manifest anxiety score questionnaire 35 failed to show significant association with CSX, Asbury et al. [25] observed that CSX patients showed more life interference and higher HADS anxiety scores than CHD patients, and CSX and CHD patients both had higher depression scores than the healthy controls $[24,25]$. Consistent with the literature, in this study, CSX patients had much higher anxiety and depression scores than the healthy controls $(p<0.001, p=0.003$ respectively). This finding suggested the role of adrenergic system hyperactivity induced by emotional disturbances in CSX.

The effect of gender is an important factor in which a majority of studies suggested that CSX occurs most commonly in females, especially in postmenopausal period, which may be explained by sex differences in hormonal status, pain perception, vulnerability, stress response, and/or coping strategies. Our study confirms that women with CSX have higher levels of anxiety, depression scores, and experience of stressful life events. The present study also found men with CSX had higher anxiety scores. Ketterer et al. [26] examined traditional risk fac-

Anxiety and Depression in CSX tors and various indices of emotional distress in males with positive angiogram, CSX, and healthy controls. They found that males with CSX reported more depression on the Ketterer Stress Symptom Frequency Checklist than positive angiographic patients and they were more likely to be spouse/friend-reported depressed and anxious on the Ketterer Stress Symptom Frequency Checklist, have higher Framingham Type A Scale scores, and reported more unprovoked nocturnal awakening compared with healthy controls. Our findings verify their results; however, men did not show higher depression scores and experience of stressful life events than healthy men group. This may be explained by the degree of stress and the reaction of males, which can be influenced by various factors such as greater effect of sympathetic nervous system and the hypothalamic-pituitaryadrenal axis, personal characteristics, sociodemographic, and cultural and occupational variables [27].

We found a negative association between educational level and experience of stressful life events among CSX patients. Patients with lower educational status had more anxiety scores and experienced stressful events $(p=0.03)$. Previous studies have revealed consistent results on the relationships between perceived stress and education level and support our findings [27]. Least educated women tend to be Type B, angry, pessimistic, depressed, and dissatisfied with their work, precluded from social support and self-esteem [28]. They have significantly higher rates of cigarette smoking, hypertension, serum cholesterol, body mass index, and coronary artery disease. This situation induces psychological and physiological stress. It is obvious that educational status influences people in terms of access to information about their health, capability of recognition and interpretation of symptoms, and the level of proficiency to discover solutions and cope with the stress.

Limitations of this study include the relatively small sample size from a single center; therefore, our findings may not be attributable to the general population. Stressors were not measured using a written stressful life events questionnaire, which may include questions on home, sexual and daily life, financial status, social and family problems, personal conflicts, job insecurity and job conflicts, educational concerns, loss and separation, and health concerns. Instead, a questionnaire on threatening experiences, including 12 life events, was administered verbally. Also, men may report fewer stressful events because they tend to avoid confessing problems. Additionally, HADS could be assessed in patients with confirmed coronary artery disease, $\geq 50 \%$ stenosis in a minimum of 
one coronary artery, as a third group. Comparisons in the levels of anxiety and depression before and after coronary angiography among groups may provide more accurate results.

\section{Conclusion}

In conclusion, HADS is a simple, cost-free tool for basic and immediate evaluation of the patient's emotional status. As HADS has good internal consistency and a 2-factor structure, the HADS subscales may provide in- formation about the contribution of anxiety or depression to the development of CSX and their role in interdisciplinary treatment strategy. Interventions targeted toward to improve quality of life and psychological support may be beneficial particularly for women and people with less education.

\section{Disclosure Statement}

The authors declare no potential conflicts of interest with respect to the research, authorship, and/or publication of this article.

\section{References}

1 Kemp HG, Kronmal RA, Vlietstra RE, Frye RL. Seven year survival of patients with normal or near normal coronary arteriograms: a CASS registry study. J Am Coll Cardiol. 1986 Mar;7(3):479-83.

2 Bass C, Mayou R. Chest pain. BMJ. 2002 Sep; 325(7364):588-91.

3 Kemp HG Jr. Left ventricular function in patients with the anginal syndrome and normal coronary arteriograms. Am J Cardiol. 1973 Sep;32(3):375-6.

4 Agrawal S, Mehta PK, Bairey Merz CN. Cardiac Syndrome X: update. Heart Fail Clin. 2016 Jan;12(1):141-56

5 Agrawal S, Mehta PK, Bairey Merz CN. Cardiac Syndrome X: update 2014. Cardiol Clin. 2014 Aug;32(3):463-78.

6 Liu A, Wijesurendra RS, Liu JM, Forfar JC, Channon KM, Jerosch-Herold M, et al. Diagnosis of microvascular angina using cardiac magnetic resonance. J Am Coll Cardiol. 2018 Mar;71(9):969-79.

7 Villano A, Lanza GA, Crea F. Microvascular angina: prevalence, pathophysiology and therapy. J Cardiovasc Med (Hagerstown). 2018 Feb;19 Suppl 1:e36-9.

8 Kaski JC. Syndrome X: A Heterogeneous Syndrome. Historical Background, Clinical Presentation, Electrocardiographic Features, And Rational Patient Management. An Overview; Angina pectoris with normal coronary arteries: Syndrome X. Springer; 1994. pp. 1-18.

9 Cunningham C, Brown S, Kaski JC. Effects of transcendental meditation on symptoms and electrocardiographic changes in patients with cardiac syndrome X. Am J Cardiol. 2000 Mar; 85(5):653-5.

10 Piegza M, Pudlo R, Badura-Brzoza K, Hese RT. [Cardiac syndrome X from a psychosomatic point of view]. Psychiatr Pol. 2008 MarApr;42(2):229-36.

11 Christoph M, Christoph A, Dannemann S, Poitz D, Pfluecke C, Strasser RH, et al. Mental symptoms in patients with cardiac symptoms and normal coronary arteries. Open Heart. 2014 Oct;1(1):e000093.
12 Cox ID, Hann CM, Kaski JC. Low dose imipramine improves chest pain but not quality of life in patients with angina and normal coronary angiograms. Eur Heart J. 1998 Feb;19(2): 250-4.

13 Cannon RO 3rd, Quyyumi AA, Mincemoyer $\mathrm{R}$, Stine AM, Gracely RH, Smith WB, et al. Imipramine in patients with chest pain despite normal coronary angiograms. N Engl J Med. 1994 May;330(20):1411-7.

14 Brugha T, Bebbington P, Tennant C, Hurry J. The List of Threatening Experiences: a subset of 12 life event categories with considerable long-term contextual threat. Psychol Med. 1985 Feb;15(1):189-94.

15 Aydemir Ö, et al. Validity and reliability of Turkish version of Hospital Anxiety and Depression Scale. Turk Psikiyatr Derg. 1997;8: 280-7.

16 Pitts WR, Lange RA, Cigarroa JE, Hillis LD. Repeat coronary angiography in patients with chest pain and previously normal coronary angiogram. Am J Cardiol. 1997 Oct;80(8): 1086-7.

17 Cox ID, Schwartzman RA, Atienza F, Brown SJ, Kaski JC. Angiographic progression in patients with angina pectoris and normal or near normal coronary angiograms who are restudied due to unstable symptoms. Eur Heart J. 1998 Jul;19(7):1027-33.

18 Sicari R, Palinkas A, Pasanisi EG, Venneri L, Picano E. Long-term survival of patients with chest pain syndrome and angiographically normal or near-normal coronary arteries: the additional prognostic value of dipyridamole echocardiography test (DET). Eur Heart J. 2005 Oct;26(20):2136-41.

19 Gulati M, Cooper-DeHoff RM, McClure C, Johnson BD, Shaw LJ, Handberg EM, et al. Adverse cardiovascular outcomes in women with nonobstructive coronary artery disease: a report from the Women's Ischemia Syndrome Evaluation Study and the St James Women Take Heart Project. Arch Intern Med. 2009 May; 169(9): 843-50.
20 Jespersen L, Hvelplund A, Abildstrøm SZ, Pedersen F, Galatius S, Madsen JK, et al. Stable angina pectoris with no obstructive coronary artery disease is associated with increased risks of major adverse cardiovascular events. Eur Heart J. 2012 Mar;33(6):734-44.

21 Cannon RO 3rd. The sensitive heart. A syndrome of abnormal cardiac pain perception. JAMA. 1995 Mar;273(11):883-7.

22 Rutledge T, Reis SE, Olson M, Owens J, Kelsey SF, Pepine CJ, et al.; Women's Ischemia Syndrome Evaluation (WISE). History of anxiety disorders is associated with a decreased likelihood of angiographic coronary artery disease in women with chest pain: the WISE study. J Am Coll Cardiol. 2001 Mar;37(3):780-5.

23 Altintas E, Yigit F, Taskintuna N. The impact of psychiatric disorders with cardiac syndrome $\mathrm{X}$ on quality of life: 3 months prospective study. Int J Clin Exp Med. 2014 Oct;7(10): $3520-7$.

24 Lutfi MF. Anxiety Level and Cardiac Autonomic Modulations in Coronary Artery Disease and Cardiac Syndrome X Patients. PLoS One. 2017 Jan;12(1):e0170086.

25 Asbury EA, Creed F, Collins P. Distinct psychosocial differences between women with coronary heart disease and cardiac syndrome X. Eur Heart J. 2004 Oct;25(19):1695-701.

26 Ketterer MW, Brymer J, Rhoads K, Kraft P, Kenyon L, Foley B, et al. Emotional distress among males with "syndrome X". J Behav Med. 1996 Oct;19(5):455-66.

27 Feizi A, Aliyari R, Roohafza H. Association of perceived stress with stressful life events, lifestyle and sociodemographic factors: a largescale community-based study using logistic quantile regression. Comput Math Methods Med. 2012;2012:151865.

28 Brezinka V, Kittel F. Psychosocial factors of coronary heart disease in women: a review. Soc Sci Med. 1996 May;42(10):1351-65. 